\title{
Os impactos da pandemia COVID-19 na educação das pessoas com deficiência do
}

\section{Estado de Alagoas}

The impacts of the COVID-19 pandemic on the education of people with disabilities in the State of Alagoas

Los impactos de la pandemia de COVID-19 en la educación de las personas con discapacidad en el Estado de Alagoas

\author{
Anna Carla Soares da Silva \\ ORCID: https://orcid.org/0000-0002-4440-888X \\ Universidade Federal de Alagoas, Brasil \\ E-mail: annac_silva@outlook.com \\ Thais Mendes de Lima Gomes \\ ORCID: https://orcid.org/0000-0001-7491-5877 \\ Universidade Federal de Alagoas, Brasil \\ E-mail: thais.gomes@eenf.ufal.br \\ Ivanise Gomes de Souza Bittencourt \\ ORCID: https://orcid.org/0000-0002-9416-3964 \\ Universidade Federal de Alagoas, Brasil \\ E-mail: ivanise.gomes@eenf.ufal.br \\ Thaynara Maria Pontes Bulhões \\ ORCID: https://orcid.org/0000-0003-2398-8173 \\ Universidade Federal de Alagoas, Brasil \\ E-mail: thaybulhoes@gmail.com \\ Carlos Henrique Ludwig \\ ORCID: https://orcid.org/0000-0002-8097-9380 \\ Universidade Federal de Alagoas, Brasil \\ E-mail: ch-ludwig@hotmail.com \\ Vitória Paulo Simplício \\ ORCID: https://orcid.org/0000-0001-5222-993X \\ Universidade Federal de Alagoas, Brasil \\ E-mail: vitória.simplicio15@gmail.com \\ Richaelle Moreira Dantas da Silva \\ ORCID: https://orcid.org/0000-0002-0087-8046 \\ Universidade Federal de Alagoas, Brasil \\ E-mail: richaelle.silva@eenf.ufal.br \\ Rayssa Francielly dos Santos Alves \\ ORCID: https://orcid.org/0000-0002-2992-7438 \\ Universidade Estadual de Ciências da Saúde de Alagoas, Brasil \\ E-mail: rayssa.alves@academico.uncisal.edu.br \\ Selma Sabrina de Albuquerque Calheiros \\ ORCID: https://orcid.org/0000-0002-8350-4410 \\ Universidade Federal de Alagoas, Brasil \\ E-mail: sabrinaalbuquerquec@hotmail.com \\ Marília Vieira Cavalcante \\ ORCID: https://orcid.org/0000-0003-0516-9019 \\ Universidade Federal de Alagoas, Brasil \\ E-mail: mariliavcavalcante@outlook.com
}

\section{Resumo}

Este estudo analisou os impactos da pandemia COVID-19 na educação das pessoas com deficiência do Estado de Alagoas, a partir da aplicação de um questionário online, sendo de natureza quantitativa e descritiva. O questionário online, desenvolvido no google forms, foi organizado em dois direcionamentos: um específico para a Pessoa com Deficiência e outro para o familiar ou responsável pela Pessoa com Deficiência, caso não fosse possível de ser respondido pela Pessoa com Deficiência. O questionário foi disponibilizado através de um link em redes sociais (Facebook, Instagram, WhatsApp) ou via e-mail para o alcance das pessoas com deficiência do Estado de Alagoas. A pesquisa foi realizada no período de 28 de agosto a 31 de outubro de 2020 com adesão de 139 participantes, sendo 36 Pessoas com Deficiência e 103 familiares ou responsáveis pela Pessoa com Deficiência que responderam ao questionário online. Diante disso, esta pesquisa possibilitou identificar os impactos na educação das Pessoa com 
Deficiência devido a pandemia COVID-19, pois, embora as atividades educacionais tenham prosseguido de forma remota, estas, segundo os participantes, não ocorreram de forma satisfatória, trazendo reflexões acerca do processo adotado pelas instituições e as necessidades de estratégias inclusivas e efetivas de escolarização, a fim de diminuir as desigualdades perante o cenário pandêmico e para além deste. As especificidades da pessoa com deficiência acrescentam características que precisam ser consideradas na construção de um plano educacional individual, porém sempre na perspectiva da inclusão e interação desses indivíduos na dinâmica escolar.

Palavras-chave: Pessoa com deficiência; Coronavírus; Educação; Ensino.

\begin{abstract}
This study the impacts of the COVID-19 pandemic on the education of people with disabilities in the State of Alagoas, from the application of an online questionnaire, being quantitative and descriptive in nature. The online questionnaire, developed in google forms, was organized in two directions: one specific for the Person with Disabilities and another for the family member or person responsible for the Person with Disabilities, if it was not possible to be answered by the Person with Disabilities. The questionnaire was made available through a link on social networks (Facebook, Instagram, Whatsapp) or via email to reach people with disabilities in the State of Alagoas, The survey was conducted from August 28 to October 31, 2020 with the support of 139 participants, 36 people with disabilities and 103 relatives or guardians of the Person with Disabilities who answered the people with disabilities due to the COVID-19 pandemic, because, although educational activities have continued remotely these, according to the participants, did not occur satisfactorily, bringing reflections about the process adopted by the institutions and the needs of inclusive and effective strategies of schooling in order to reduce inequalities in the pandemic scenario and beyond this. The specificities of people with disabilities add characteristics that need to be considered in the construction of an individual educational plan, but always from the perspective of the inclusion and interaction of these individuals in the school dynamics.
\end{abstract}

Keywords: Disabled person; Coronavirus; Education; Teaching.

\title{
Resumen
}

Este estudio analizó los impactos de la pandemia de COVID-19 en la educación de las personas con discapacidad en el Estado de Alagoas, a partir de la aplicación de un cuestionario em línea, siendo de carácter cuantitativo y descriptivo. El cuestionario em línea, desarrollado en formularios de google, se organizó em dos direcciones: una específica para la Persona con Discapacidad y otra para el familiar o persona responsable de la Persona con Discapacidad, si no era posible ser respondida por la Persona con Discapacidad. El cuestionario se puso a disposición a través de un enlace en redes sociales (Facebook, Instagram, Whatsapp) o vía correo electrónico para llegar a personas con discapacidad en el Estado de Alagoas. La encuesta se realizó del 28 de agosto al 31 de octubre de 2020 con el apoyo de 139 participantes, 36 personas con discapacidad y 103 familiares o tutores de la persona con Discapacidad que respondieron el cuestionario en línea. Por lo tanto, esta investigación ha logrado identificar los impactos en la educación de las personas con discapacidad debido a la pandemia de COVID-19, ya que, si bien las actividades educativas han continuado de manera remota, estas, según los participantes, no ocurrieron satisfactoriamente, trayendo reflexiones sobre el proceso adoptado por las instituciones t las necesidades de estrategias inclusivas y efectivas de escolarización, con el fin de reducir las desigualdades en el escenario de pandemia y más allá de este. Las especificidades de las personas con discapacidad suman características que deben ser consideradas en la construcción de un plan educativo individual, pero siempre desde la perspectiva de la inclusión e interacción de estos individuos en la dinámica escolar.

Palabras clave: Persona con discapacidad; Coronavirus; Educación; Enseñanza.

\section{Introdução}

Em 31 de dezembro de 2019, a Organização Mundial da Saúde (OMS) foi notificada sobre um aumento de casos de pneumonia na cidade de Wuhan, província de Hubei, localizada na China. Posteriormente, foi identificado que se tratava de uma nova cepa de coronavírus (Organização Pan-Americana da Saúde [OPAS], 2021; Souto, 2020).

Em 11 de março de 2020, a OMS declarou que o novo coronavírus (SARS-CoV-2), causador da doença COVID-19, passou de uma epidemia para o estágio de pandemia. Tal decisão decorreu do aumento exponencial do número de casos mundialmente. Como estratégia de limitar a propagação do vírus e conter esta pandemia, as autoridades sanitárias aderiram ao isolamento social (OPAS, 2021).

Nesse contexto, houve uma mudança abrupta na rotina da sociedade, afetando consideravelmente a sua dinâmica, principalmente no que concerne ao distanciamento social, fechamento das instituições de educação e mantendo somente os serviços considerados essenciais à sobrevivência, abastecimento, saúde e segurança da população (Bonaparte, Santos, Santos, 
Santos \& Haiachi, 2020).

No que tange às Pessoas com Deficiência (PcD), esse período de pandemia acabou por elevar ainda mais os seus desafios, principalmente os que remetem a educação e saúde, bem como os aspectos biopsicossociais (Coura \& Almeida, 2020).

Diante disso, instalou-se uma crise humanitária com impactos na esfera das políticas educacionais para este período de isolamento social, no âmbito Municipal, Estadual e Federal. As medidas adotadas para este período, foram o uso de meios não presenciais, como: plataformas digitais e virtuais, mídias sociais, vídeo aulas, vídeos educativos, entre outros, assim como a mediação dos cuidadores para a realização das atividades escolares (Souza \& Dainez, 2020).

O Conselho Nacional de Educação (CNE) com o propósito de orientar a integração curricular e a prática das ações educacionais em nível nacional, emitiu o Parecer CNE/CP N 11 , de 7 de julho de 2020. Este refere-se a orientações educacionais para a realização de aulas e atividades pedagógicas presenciais e não presenciais no contexto da pandemia (Brasil, 2020a). Além disso, em 18 de agosto, foi sancionada a Lei $\mathrm{n}^{\circ}$ 14.040/2020, que estabeleceu normas educacionais excepcionais a serem adotadas durante o estado de calamidade pública (Brasil, 2020b).

A Constituição Federal (Brasil, 1988), a Convenção Internacional sobre os Direitos da Pessoa com Deficiência da ONU (Brasil, 2009) e a Lei Brasileira de Inclusão no 13.146/2015 (Brasil, 2015), garantem o direito à educação inclusiva. Em relação ao contexto da pandemia, o Parecer $\mathrm{n}^{\circ} 11$ do CNE, estabeleceu a necessidade de ofertar continuidade a esse direito, como também, qualidade e equidade (Matuoka, 2020).

Entretanto, apesar da existência dessas leis, foram inúmeras as desigualdades enfrentadas no direito à educação e inclusão das pessoas com deficiência nos trabalhos pedagógicos remotos, como também, problemas de acesso às tecnologias de informação e comunicação, e a falta de suporte ofertada aos familiares pelas instituições de ensino para a realização das atividades escolares (Matuoka, 2020).

Em Alagoas, segundo dados do Censo de 2010 (Brasil, 2012), 859.515 pessoas possuem algum tipo de deficiência. Dado os desafios que se apresentaram, este artigo teve como objetivo analisar os impactos da pandemia do COVID-19 na educação das PcD do Estado de Alagoas, a partir de um questionário online realizado com estas ou com seus familiares.

\section{Metodologia}

Trata-se de um estudo com abordagem quantitativa e descritiva, com a população de Pessoas com Deficiência do Estado de Alagoas. Nesse sentido, Pereira e Ortigão (2016) afirmam que as pesquisas quantitativas são indicadas para reconhecer determinadas características em certa população, a partir do seu grau e abrangência.

Foi aprovado pelo Comitê de Ética em Pesquisa da Universidade Federal de Alagoas (UFAL), sob o Parecer $\mathrm{n}^{\circ}$ 4.198.127 e CAAE nº 34107320.3.0000.5013 e foram respeitados os procedimentos éticos, estabelecidos na Resolução 466/12 e 510/16.

A coleta de dados se deu através de um questionário online desenvolvido no google forms, dividido em dois direcionamentos: um específico para a PcD, e outro especificamente para o familiar ou Responsável pela Pessoa com Deficiência (R-PcD), caso não fosse possível de ser respondido pela PcD.

O questionário foi disponibilizado por um link em redes sociais (Facebook, Instagram, WhatsApp) ou via e-mail para o alcance das PcD do Estado de Alagoas. Ao selecionarem o link disponibilizado, imediatamente os interessados tiveram acesso ao Termo de Consentimento Livre e Esclarecido (TCLE), com todas as informações sobre o estudo e um canal de comunicação com os pesquisadores. 
A pesquisa foi realizada no período de 28 de agosto a 31 de outubro de 2020 com adesão de 139 participantes, sendo 36 Pessoas com Deficiência e 103 familiares ou Responsáveis pela Pessoa com Deficiência que responderam ao questionário online. As respostas foram transportadas para o Microsoft Excel para análise e descrição dos dados.

\section{Resultados}

Os dados obtidos neste estudo foram organizados a partir de três unidades temáticas: 1. Perfil dos Participantes: Pessoas com Deficiência e Familiares/Responsáveis das Pessoas com Deficiência; 2. Educação das Pessoa com Deficiência no Estado de Alagoas e 3. Dados Familiares/Residenciais: Pessoas com Deficiência e Familiares/Responsáveis das Pessoas com Deficiência, as quais apresentam os resultados da pesquisa.

\subsection{Perfil dos Participantes: Pessoas com Deficiência e Familiares/Responsáveis das Pessoas com Deficiência}

O sexo biológico dos Responsáveis das Pessoas com Deficiência (R-PcD) foi predominentemente Feminino (87,4\%), enquanto o das Pessoas com Deficiência (PcD) sob os seus cuidados configurou-se principalmente em Masculino (67,96\%). O sexo biológico das PcD que responderam ao questionário foi feminino $(63,9 \%)$ e masculino $(36,1 \%)$, representando uma predominância de pessoas do sexo feminino na adesão em responderem ao questionário da pesquisa.

Os R-PcD, em sua maioria, declararam que não apresentavam nenhum tipo de deficiência, enquanto que 1,9\% tinham Síndrome de Down, $1 \%$ Transtorno do Espectro Autista e 1\% Deficiência Visual. E mencionaram que as PcD sob os seus cuidados apresentavam as seguintes deficiências: Transtorno do Espectro Autista (43,6\%), Síndrome de Down (15,4\%), Intelectual (3,9\%), Transtorno do Espectro Autista e TDAH (3,9\%), Auditiva, Intelectual e Física, Paralisia Cerebral, Paralisia Cerebral e TEA, Surdez Neurosensorial e Hidrocefalia, Visual, Intelectual e Motora (1,9\%), Deficiência Auditiva e TEA, Deficiência Moderada, Esquizofrenia Paranóide, Física, Hidrocefalia Compensada, Intelectual e Retardo Mental, Mental e visual, Microcefalia, Mielomeningocele (1\%). Enquanto que as PcD respondentes ao questionário, conforme consta na Tabela 1, apresentavam as deficiências: Física (50\%), Visual (22,2\%), Auditiva (16,7\%), TEA (5,6\%), Múltipla, Síndrome de Farh e Transtorno de Déficit de Atenção (2,8\%).

Tabela 1: Tipo de deficiência dos participantes com deficiência do estudo no Estado de Alagoas, Brasil, 2020.

\begin{tabular}{cccc}
\hline Variável & & Resultados \\
\hline & Física & $\mathbf{n}$ & \% \\
& Visual & 18 & $50 \%$ \\
& Auditiva & 7 & $22,2 \%$ \\
Tipo de deficiência & 6 & $16,7 \%$ \\
& Intelectual & 4 & $7 \%$ \\
& Múltipla & 1 & $4,1 \%$ \\
& TOTAL & 36 & $100 \%$ \\
\hline
\end{tabular}

Fonte: Autores (2020).

As idades das PcD e dos R-PcD que fizeram parte desta pesquisa, estão apresentadas a seguir na Tabela 2: 
Tabela 2: Idade das Pessoas com Deficiência e dos Responsáveis das Pessoas com Deficiência participantes desta pesquisa.

\begin{tabular}{|c|c|c|c|}
\hline \multirow[t]{2}{*}{ Variável } & & \multicolumn{2}{|c|}{ Resultados } \\
\hline & & n & $\%$ \\
\hline \multirow{10}{*}{ Idade da PcD } & 20 a 24 anos & 1 & $2,8 \%$ \\
\hline & 25 a 29 anos & 6 & $16,7 \%$ \\
\hline & 30 a 34 anos & 6 & $16,7 \%$ \\
\hline & 35 a 39 anos & 6 & $16,7 \%$ \\
\hline & 40 a 44 anos & 3 & $8,3 \%$ \\
\hline & 45 a 49 anos & 6 & $16,7 \%$ \\
\hline & 50 a 54 anos & 3 & $8,3 \%$ \\
\hline & 55 a 59 anos & 1 & $2,8 \%$ \\
\hline & 60 anos ou mais & 4 & $11 \%$ \\
\hline & \multirow[t]{2}{*}{ TOTAL } & 36 & $100 \%$ \\
\hline \multirow{12}{*}{ Idade do R-PcD } & & n & $\%$ \\
\hline & 18 a 19 anos & 1 & $1 \%$ \\
\hline & 20 a 24 anos & 5 & $4,9 \%$ \\
\hline & 25 a 29 anos & 12 & $11,7 \%$ \\
\hline & 30 a 34 anos & 13 & $12,6 \%$ \\
\hline & 35 a 39 anos & 17 & $16,5 \%$ \\
\hline & 40 a 44 anos & 22 & $21,4 \%$ \\
\hline & 45 a 49 anos & 16 & $15,5 \%$ \\
\hline & 50 a 54 anos & 6 & $5,8 \%$ \\
\hline & 55 a 59 anos & 2 & $1,9 \%$ \\
\hline & 60 anos ou mais & 9 & $8,7 \%$ \\
\hline & TOTAL & 103 & $100 \%$ \\
\hline
\end{tabular}

Fonte: Elaborado pelos autores (2020).

Os participantes da pesquisa, residiam, em sua maioria em zona urbana, e em zona rural aproximadamente apenas $2 \%$, sendo em Maceió (73,5\%), Penedo (5,5\%), Delmiro Gouveia (4,35\%), Arapiraca (4,2\%), Palmeira dos Índios (3,3\%), Pilar $(2,75 \%)$, Marechal Deodoro (1,4\%); os $5 \%$ dos demais estavam distribuídos nas seguintes cidades (Coité do Nóia, Barra de São Miguel, Campo Alegre, Coruripe, Igaci, Junqueiro, Paripueira, Piranhas e Rio Largo), e representando entre si cerca de $1 \%$, de acordo com o que se apresenta na Tabela 3 : 
Tabela 3: Distribuição demográfica dos participantes do estudo no Estado de Alagoas, Brasil, 2020.

\begin{tabular}{cccc}
\hline Variável & & Resultados & \% \\
& & $\mathbf{n}$ & $73,5 \%$ \\
& Maceió & 26 & $5,5 \%$ \\
& Penedo & 2 & $4,35 \%$ \\
Municípios dos & Delmiro Gouveia & 2 & $4,2 \%$ \\
participantes & Arapiraca & 2 & $3,3 \%$ \\
& Palmeira dos Índios & 2 & $2,75 \%$ \\
& Pilar & 1 & $1,4 \%$ \\
& Marechal Deodoro & 1 & $5 \%$ \\
& Outros municípios & 2 & $100 \%$ \\
\hline
\end{tabular}

Fonte: Elaborado pelos autores (2020).

Com relação a classificação da cor ou raça, as PcD se declararam: Branco(a) (52,8\%), Pardo(a) (33,3\%) e Negro(a) (13,9\%). Já os R-PcD, consideraram-se: Pardo(a) (50,5\%), Branco(a) (35,9\%) e Negro(a) (13,6\%).

No que tange o estado civil, os R-PcD eram 60,2\% casados(a), 20,4\% solteiros(a), 10,7\% estavam em união estável e 8,7\% divorciados(a), entretanto as PcD eram em sua maioria solteiros(a), correspondendo a 47,2\%, 33,3\% casados(a), 13,9\% em união estável e 5,6\% divorciados(a).

\subsection{Educação das Pessoa com Deficiência no Estado de Alagoas}

Sobre a educação, as PcD afirmaram, conforme consta na Tabela 4, que tinham: Ensino Fundamental 1 (do $1^{\circ}$ ao $5^{\circ}$ ano), Completo (8,3\%), Ensino Médio Incompleto (5,6\%), Ensino Médio Completo (19,4\%), Ensino Superior Incompleto $(11,1 \%)$, Ensino Superior Completo (27,8\%), Especialização (25\%) e Doutorado (2,8\%). 
Research, Society and Development, v. 10, n. 15, e425101523086, 2021

(CC BY 4.0) | ISSN 2525-3409 | DOI: http://dx.doi.org/10.33448/rsd-v10i15.23086

Tabela 4: Escolaridade dos participantes deste estudo no Estado de Alagoas, Brasil, 2020.

\begin{tabular}{|c|c|c|c|}
\hline \multirow[t]{2}{*}{ Escolaridade } & & \multicolumn{2}{|c|}{ Resultados } \\
\hline & & $\bar{n}$ & $\%$ \\
\hline \multirow[t]{10}{*}{ Escolaridade PcD } & $\begin{array}{c}\text { Ensino Fundamental I (do } 1^{\circ} \\
\text { ao } 5^{\circ} \text { ano) completo }\end{array}$ & 3 & $8,3 \%$ \\
\hline & Ensino Médio Incompleto & 2 & $5,6 \%$ \\
\hline & Ensino Médio Completo & & \\
\hline & & 7 & $19,4 \%$ \\
\hline & & 4 & $11,1 \%$ \\
\hline & Ensino Superior Incompleto & & \\
\hline & & 10 & $27,8 \%$ \\
\hline & Ensino Superior Completo & 10 & $27,8 \%$ \\
\hline & Especialização & 36 & $100 \%$ \\
\hline & TOTAL & & \\
\hline \multirow{12}{*}{$\begin{array}{l}\text { Escolaridade segundo o R- } \\
\text { PcD }\end{array}$} & Analfabeto & 13 & $12,6 \%$ \\
\hline & $\begin{array}{l}\text { Educação Infantil (maternal } \\
\text { ao jardim 2) }\end{array}$ & 39 & $37,9 \%$ \\
\hline & $\begin{array}{l}\text { Ensino Fundamental I (do } 1^{\circ} \\
\text { ao } 5^{\circ} \text { ano) incompleto }\end{array}$ & 27 & $26,1 \%$ \\
\hline & $\begin{array}{l}\text { Ensino Fundamental I (do } 1^{\circ} \\
\text { ao } 5^{\circ} \text { ano) completo }\end{array}$ & 2 & $1,9 \%$ \\
\hline & Ensino Fundamental II (do $6^{\circ}$ & 6 & $5,8 \%$ \\
\hline & ao $9^{\circ}$ ano) incompleto & 1 & $1 \%$ \\
\hline & $\begin{array}{l}\text { Ensino Fundamental II (do } \\
06^{\circ} \text { ao } 09^{\circ} \text { ano) completo }\end{array}$ & 5 & $4,9 \%$ \\
\hline & Ensino Médio Incompleto & 5 & $4,9 \%$ \\
\hline & Ensino Médio Completo & 5 & $4,9 \%$ \\
\hline & Ensino Superior Completo & & \\
\hline & & 103 & $100 \%$ \\
\hline & TOTAL & & \\
\hline
\end{tabular}

Fonte: Elaborado pelos autores (2020).

Os R-PcD responderam que antes da pandemia não estavam estudando, correspondendo a um percentual de $87,4 \%$, já $12,6 \%$ afirmaram que estavam estudando. Enquanto que, as $\mathrm{PcD}$ sob um percentual de $61,1 \%$ responderam que estavam estudando e $38,9 \%$ não estavam.

Já quanto à continuidade dos estudos durante a pandemia de forma remota (em casa), os R-PcD declaram: 56,3\% que estavam estudando e 43,7\% não estavam. As PcD 55,6\% continuaram estudando e 44,4\% não deram continuidade aos estudos.

No tocante às instituições de ensino a qual pertenciam, os R-PcD declararam que as PcD sob os seus cuidados, faziam parte $63,1 \%$ da rede privada, 35,9\% da rede pública e $1 \%$ nunca estudou/nunca estudaram. As PcD disseram que 55,6\% estudavam em rede privada e $44,4 \%$ em rede pública. 
Ademais, quanto às instituições de ensino que permaneceram com as atividades de forma remota durante a pandemia Covid-19, os R-PcD informaram que 70,9\% das PcD deram seguimento às atividades, 20,4\% não continuaram. As PcD disseram que $55,9 \%$ prosseguiram e $44,1 \%$ não o fizeram.

Além disso, quando questionados se as atividades propostas para a continuidade do ensino pelas instituições a qual faziam parte, foram satisfatórias, as PcD responderam que: 72,2\% não foram satisfatórias e 27,8\% foram satisfatórias.

\subsection{Dados Familiares/Residenciais: Pessoas com Deficiência e Familiares/Responsáveis das Pessoas com Deficiência}

Os R-PcD responderam que, além deles, ainda residem em suas casas quatro ou mais pessoas, obtendo um percentual de 49,5\%, três pessoas 42,7\% e duas pessoas 7,8\%. Já as PcD alegaram morarem sozinhas 2,8\%, com uma pessoa 5,6\%, duas pessoas $(25 \%)$, três pessoas $(16,7 \%)$ e quatro ou mais pessoas $50 \%$.

Além disso, os R-PcD também afirmaram que preponderantemente residiam na sua casa uma $\mathrm{PcD}$, correspondendo a $93,2 \%$, duas pessoas $3,9 \%$ e nenhuma $2,9 \%$. As PcD disseram que $52,8 \%$ da sua família, não apresenta deficiência e $47,2 \%$ disseram que apresentam algum tipo de deficiência.

Paradoxalmente, os R-PcD declararam apresentar os seguintes vínculos com a PcD sob os seus cuidados: sobretudo 78,6\% eram mães, 5,9\% pais, 4,9 filhos(a), 2,9\% irmãso(a), 2,9\% avós(ô), 2,9\% sem laço de parentesco e 1,9\% eram tios.

No que tange ao diagnóstico de COVID-19 das pessoas que moravam com a PcD, os R-PcD disseram que 86,4\% não foram diagnosticados e 13,6\% foram diagnosticados. As PcD relataram que: Ninguém foi Diagnosticado com COVID-19 $83,3 \%$, Irmão(s) 8,3\%, Mãe 5,6\%, Eu 2,8\%, Pai 2,8\% e Meu Filho 2,8\%.

\section{Discussão}

O perfil analisado a partir do resultado da pesquisa, permitiu o cruzamento dos dados que estão contidos na Cartilha do Censo 2010 - Pessoas com Deficiência, publicados em 2012 pela Secretaria de Direitos Humanos (SDH/PR). Segundo o arquivo, 23,9\% do total da população brasileira apresenta pelo menos um tipo de deficiência, sendo estes majoritariamente do sexo feminino (26,5\% do total). Destes, a maioria apresenta deficiência visual $(18,6 \%)$, deficiência física $(7 \%)$, auditiva $(5,1 \%)$ e intelectual (1,4\%). Não há menções de deficiências múltiplas (Brasil, 2012).

No Nordeste, existem cerca de 26,3\% de Pessoas com Deficiência, das quais Alagoas fica em antepenúltimo lugar com menor número de $\mathrm{PcD}$, representando cerca de 27,5\% do total brasileiro (Brasil, 2012). Alagoas, segundo a pesquisa, segue o mesmo padrão demográfico da Cartilha, segundo a variável sexo (Feminino=63,9\%), porém no que se refere a "tipos de deficiência", a predominância é de deficiência física (50\%), seguido de deficiência visual $(22,2 \%)$, auditiva (16,7\%), e apresenta casos de deficiência múltipla $(4,1 \%)$.

Ainda, analisou-se que o território nacional apresenta uma porcentagem elevada de pessoas com 65 anos ou mais com deficiência (67,73\%), enquanto que apenas 25\% estão distribuídos entre 15 e 64 anos, e 7,53\% de 0 a 14 anos (Brasil, 2012). Em Alagoas, os dados destoam do território nacional, primeiramente nos dados referentes às pessoas com mais de 60 anos, que representam apenas $11,1 \%$, enquanto que a faixa etária com maior representatividade está compreendida entre 25 e 39 anos.

Segundo o Censo de 2010, a situação nacional de domicílio correspondia a 84,4\% urbano e 15,6\% rural. No que se refere a população com deficiência, essa porcentagem cai para 20,16\% urbano e 3,73\% rural (Brasil, 2012). Em Alagoas, segundo a pesquisa analisada, $98 \%$ residiam na zona urbana, enquanto que apenas $2 \%$ residiam na zona rural.

Proporcionalmente, os dados sobre a residência da população brasileira que não apresentam deficiência e com deficiência seguem o mesmo fluxo, cerca de 5 pessoas em zona urbana para cada 1 que reside em zona rural, enquanto que no Estado de Alagoas essa proporção aumenta em 10 vezes, no qual para cada 50 pessoas em zona urbana, existe apenas uma pessoa com deficiência em zona rural. 
De acordo com Censo de 2010, o índice de analfabetismo diminuiu de 11,15\% para 7,5\% num espaço-tempo de 10 anos; em Alagoas, essa taxa era de 22,5\% na população de 10 ou mais anos de idade. Quando levamos em consideração à PcD, a taxa de alfabetização era de $81,7 \%$, nas quais as regiões Nordeste e Norte apresentaram os menores índices de alfabetização, ainda que esses termos sejam menores do que a alfabetização da população geral em qualquer região do Brasil.

Quanto ao nível de instrução, o referente a PcD é de 14,2\% para o Ensino Fundamental Completo (EFC), 17,7\% para o Ensino Médio Completo (EMC) e 6,7\% para o Ensino Superior Completo (ESC). Pessoas com deficiência sem instrução ou Ensino Fundamental Incompleto (EFI) eram mais do que 38,2\%, número muito menor que o da população em geral, que eram de mais de $60 \%$ (Brasil, 2012).

Segundo a pesquisa, em Alagoas, a população da PcD sem instrução ou com EFI está abaixo da média de 2010 $(12,6 \%)$. Segundo o R-PcD, estão abaixo da média nacional, a taxa de analfabetos (12,6\%), EFI (26,1\%), EMC (4,9\%), e ESC $(4,9 \%)$. Entretanto, esses valores se invertem quando levados em consideração somente as respostas das próprias PcD, nos quais as taxas de ESC e Especialização atingem quase $28 \%$ cada, EMC acima de 19\%, e somente EFC abaixo da média nacional (2010), com 8,3\%.

O estabelecimento do isolamento social, como maneira de contenção da disseminação da COVID-19, evidenciou a necessidade de estratégias para o retorno e a manutenção das atividades educacionais, de modo que as medidas implementadas visavam minimizar os prejuízos já estabelecidos sob a educação. Em Alagoas, a suspensão das atividades educacionais, rede pública e privada, foi estabelecida em 17 de março de 2020, de acordo com o Decreto nº 69.527 (Alagoas, 2020).

A Medida Provisória no 936/2020, que institui o Programa Nacional de Manutenção de Emprego e Renda, amparado pelo parecer do Conselho Nacional de Educação, aprovou as diretrizes que orientaram as instituições de ensino básico e superior no período da Pandemia COVID-19. Essas medidas se tornaram possíveis devido a Reforma do Ensino Médio (EM), em 2017, sobre o possível cumprimento da carga horária letiva total na modalidade de Educação a Distância (EAD) em 20\% para o EM regular não noturno, desde que as atividades pedagógicas sejam estabelecidas adequadamente e orientadas por docentes (Brasil, 2020c).

Em junho de 2020, como parte das medidas de prevenção à disseminação do vírus da COVID-19, a Secretaria do Estado de Alagoas estabeleceu o Regime Especial de Atividades Escolares não presenciais (REAENP) para as unidades de Ensino da Rede Pública Estadual. O REAENP, segundo sua concepção e implementação divulgada, seria desenvolvida nas 311 Unidades de Ensino Estaduais, de forma interdisciplinar para as turmas do $1^{\circ}$ ano do Fundamental a $2^{\mathrm{a}}$ série do Ensino Médio, enquanto que as turmas da $3^{\text {a }}$ série do Ensino Médio estariam inseridas no programa Foca no Exame Nacional do Ensino Médio (ENEM). A Secretaria de Educação (SEDUC) organizou salas virtuais de aprendizagem e ferramentas complementares para contato e mobilização dos estudantes, bem como materiais físicos para os alunos que não tinham acesso ao meio digital.

O Parecer DCE/CD n5/2020 estabeleceu a reorganização do calendário escolar, ofertando atividades não presenciais, com o objetivo de cumprir com a carga horária anual letiva necessária e estabelecida pelo MEC. Ainda, deveriam ser observadas as particularidades dentro da rede educacional, bem como os limites relacionados ao acesso dos estudantes e da própria rede às tecnologias necessárias e disponíveis para continuidade do ensino. Propostas inclusivas devem ser consideradas, visando a diminuição das desigualdades e diferentes oportunidades educacionais (Brasil, 2020d).

É importante destacar que nenhuma das legislações acima citadas fazem menção e/ou referência a Educação Inclusiva, sendo esta, respaldada pela Lei de Diretrizes e Bases da Educação Nacional no 4.024 (Brasil, 1961), que em setembro de 2020 passou a ser instituída pelo Decreto n 10.502- sobre a Política Nacional de Educação Especial (Brasil, 2020e). Em nenhum momento durante a Pandemia COVID-19 essas políticas deixaram de ter validade.

A pesquisa realizada aponta que, cerca de $75 \%$ das PcD estavam inseridas em redes de ensino antes da determinação de pandemia, em janeiro de 2020; cerca de 55\% dos que já frequentavam a escola, pertenciam à rede privada de ensino. As 
atividades remotas, como previsto, foram continuadas durante a pandemia, e se estabeleceram em $63 \%$ das instituições avaliadas.

É necessário destacar que existem dois pontos que precisam ser discutidos: o primeiro deles, refere-se então a dificuldade da efetivação da educação inclusiva em escolas de ensino regular. Por consequência, fazia-se necessário a adaptação desse ensino inclusivo para com a pessoa com deficiência na modalidade EAD, buscando o que já é previsto em Lei, e descrito em vários documentos auxiliares do Governo Federal e Estaduais. O outro, é sobre a necessidade de adaptação ao ensino na modalidade remota, de acordo com as necessidades de cada indivíduo e sua respectiva deficiência. A discussão desse ponto não se faz para reduzir um indivíduo a sua deficiência, assim deixando de ser um ser plural, para ser apenas uma característica limitante; mas sobre o conhecimento sobre essas deficiências, entendendo que estas acrescentam a esse ser, individualidades mais específicas, que podem ser desafiadoras e que afetam diretamente o desenvolvimento desses. Nesse sentido Oliveira, Oliveira e Barbosa (2021) também afirmaram que a educação inclusiva está distante da realidade do ensino remoto. Em seu estudo sobre a percepção dos professores sobre a inclusão no ensino remoto dos alunos com deficiência durante a pandemia do novo coronavírus, os referidos autores apontaram que a Educação Inclusiva continuava sendo um enorme desafio a ser enfrentado e que a Pandemia do novo Coronavírus agravou o cenário de exclusão escolar para os alunos com deficiência.

Nesse contexto, partindo do princípio de que o currículo não se deve enclausurar pelas limitações de qualquer finalismo instrumental, devendo, pelo contrário, abrir-se a todas as esferas da experiência da vida humana (Morgado, Sousa \& Pacheco, 2020), e assim sendo, a discussão acerca da realidade da pessoa com deficiência inserida nesse momento de pandemia faz-se necessário, visto que, as peculiaridades do universo do cuidado à pessoa com deficiência precisam ser consideradas, mediante às medidas globais, que para se tornarem eficazes, precisam ser adaptadas a fim de responder a contextos específicos, tendo em vista a singularidade e riqueza de demandas deste grupo (Coura \& Almeida, 2020).

Com o objetivo de dar continuidade ao ano letivo, algumas instituições de ensino públicas e privadas utilizaram plataformas e canais via web para levar os conteúdos aos alunos desde a educação infantil até o ensino superior (Carvalho, 2020). O questionamento acerca da satisfação das atividades remotas estabelecidas, em sua maioria foram insatisfatórias, das quais $72 \%$ das PcD acusam e 76\% dos R-PcD, sob diversos pontos de vista. Os principais desafios relatados na pesquisa, referem-se principalmente a falta de atividades apropriadas para $\mathrm{PcD}$, dificultando o processo de ensino aprendizagem, bem como a falta de apoio pedagógico, dificuldade de uso das plataformas digitais, aumento do nível de estresse da PcD e os materiais não adaptados e específicos para cada deficiência.

As avaliações positivas referem-se ao feedback proposto pelas instituições, respeito ao tempo de exposição às telas de acordo com a idade, acompanhamento individual e a continuidade de escolarização, ainda que de maneira não apropriada.

Não deixando de voltar a reconhecer o inestimável contributo que as tecnologias disponibilizam em tempo de pandemia, garantindo que as escolas pudessem continuar a funcionar, a digitalização permanente das relações humanas poderá acabar, inevitavelmente, por desfigurar o próprio fenômeno educativo, que não se compadece de uma entrega permanente ao domínio do digital (Morgado, Sousa \& Pacheco, 2020).

Segundo Carvalho (2020), o desafio de romper com a exclusão social fez com que muitos professores precisassem se reinventar e criar estratégias de aprendizagem através do trabalho remoto, ou seja, feito à distância. O alcance geográfico é considerado, aparentemente, o que mais exemplifica a situação atual durante a quarentena, a necessidade de levar a aula até o espaço em que se encontram os alunos, quando é invertido o processo educacional.

A distância do tempo, que oportuniza ao aluno a realizar os exercícios em seu próprio ritmo, a distância tecnológica, que pode ser pensada como a oportunidade de valorizar a inclusão digital para garantir o aprendizado, assim como as 
distâncias psicossocial e socioeconômica, que ocorrem quando inexiste o ensino presencial e existe a necessidade de projetos de qualidade que possam ampliar e democratizar a oferta educacional a esses alunos (Carvalho, 2020).

Alguns relatos na pesquisa, fazem referência ao tempo de exposição a telas, aumentados devido a necessidade de continuidade da educação, ainda que alguns responsáveis mencionam como ponto positivo. A Sociedade Brasileira de Pediatria (SBP), emitiu em 2020 uma nota de alerta sobre as recomendações do uso saudável das telas digitais, voltados especificamente para a atual situação da pandemia de COVID19. Evidenciam-se que as análises feitas em relação a esse tema, precisam levar em consideração as diferenças sociais, a abrangência continental brasileira e o período de tempo avaliado.

Ainda, as sugestões da SBP englobam o estabelecimento prévio ao tempo de exposição, considerando a idade, alimentação, descanso e horário de sono; reservar um tempo para se manter o relacionamento afetivo, respeitando o distanciamento social e utilizado para comunicação com familiares, amigos e outros; estabelecer determinado número de horas-aulas e horas-estudo, de acordo com a idade, o enquadramento da escola, equipamentos utilizados e qualidade da internet; reservar um número de horas para a convivência domiciliar com outras pessoas da residência; horário específico para lazer; dialogar e buscar formas de manter a segurança desse indivíduo, sempre tendo notas sobre as autoridades responsáveis, para o caso de eventuais problemas.

\section{Considerações Finais}

Diante do exposto foi possível analisar o perfil das pessoas com deficiência do Estado de Alagoas, o retrato da educação em tempos de pandemia COVID-19 e os seus impactos para as PcD. Nesse sentido, observou-se que mesmo em contexto pandêmico, as PcD deram continuidade aos seus estudos de forma remota, sendo essas, em sua grande maioria, pertencentes às instituições privadas de ensino. Contudo, mesmo com o seguimento nas atividades educacionais, estas não ocorreram de forma satisfatória, trazendo reflexões acerca do processo adotado pelas instituições e as necessidades de estratégias efetivas de escolarização, tornando-as de fato inclusiva.

$\mathrm{O}$ reconhecimento da PcD para além da sua deficiência é o primeiro desafio a ser superado, pois o não reconhecimento produz implicações nas mais diversas áreas da vida, incluindo a continuidade no processo educacional. Preconceitos, estigmas e as fragilidades encontradas no sistema educacional para a uma educação inclusiva efetiva já eram realidades que as PcD e seus familiares enfrentavam e com a pandemia COVID-19 isso se intensificou, posto que, como forma de contenção à disseminação da COVID-19, foi adotada a modalidade de atividades escolares não presenciais para conclusão do calendário escolar. Entretanto, esta apresentou fragilidades, evidenciadas nas dificuldades das PcD reproduzirem as atividades em casa por falta de suporte e instruções para o desenvolvimento destas, além dos prejuízos em decorrência da falta de socialização e principalmente no que concerne o processo de ensino e aprendizagem desses indivíduos.

Esse estudo mostrou sobre a importância de desenvolver medidas que favoreçam a inclusão das $\mathrm{PcD}$, avaliando as suas necessidades e criando estratégias que facilitem o ensino, a fim de diminuir as desigualdades no âmbito educacional. Ainda que esteja previsto em Lei o direito à educação inclusiva para as $\mathrm{PcD}$, esse processo foi desafiador durante a pandemia COVID-19, evidenciando a necessidade de medidas efetivas de escolarização. A busca de ferramentas acessíveis para educadores, pais e/ou responsáveis, com especificidade para $\mathrm{PcD}$, pode ser realizada a partir da adaptação funcional de atividades realizadas ainda em sala de aula no ensino presencial, bem como o uso apropriado das tecnologias adotadas para o sistema de ensino.

O apoio aos responsáveis, auxílio na construção de rotinas dentro do isolamento social, bem como no desenvolvimento de metodologias para auxiliar no processo educacional da $\mathrm{PCD}$, surge como uma demanda pedagógica que necessita da medicação de professores e outros profissionais, que, atuando em conjunto, podem minimizar os danos e os impactos ocasionados pelo isolamento social sob a educação. 
Desse modo, torna-se importante o desdobramento de novas pesquisas direcionadas à educação das PcD. Trabalhos futuros que investiguem as situações envolvidas nos processos de prosseguimento ou interrupção da escolaridade para além do cenário pandêmico, pois a escolarização das PcD sempre se configurou em desafios para o sistema escolar. É necessário, inclusive, mais estudos que possibilitem as narrativas pessoais desses indivíduos que vivenciam esses desafios para a sua escolarização, pois são eles que também devem ser ouvidos sobre assuntos que lhes dizem respeito, possibilitando conhecer as suas principais necessidades que possam indicar caminhos para a sua inclusão e prosseguimento escolar.

\section{Referências}

Alagoas. (2020). Decreto $n^{\circ}$ 69.527, de 17 de março de 2020. Institui medidas temporárias de enfrentamento da emergência de saúde pública de importância internacional decorrente do covid-19 (coronavírus), no âmbito da rede pública e privada de ensino no âmbito do estado de alagoas, e dá outras providências. Maceió, Alagoas.

Bonaparte, J. P., Santos, T. N., Santos, A. C. A., Santos, J. B. S. \& Haiachi, M. (2020). Desafios do distanciamento social para pessoas com deficiência: programa desafio rotina saudável. Ambiente: Gestão e Desenvolvimento, 1(1), 25-39.

Brasil. (2020). Parecer CNE/CP $n^{\circ} 11 / 2020$, de 7 de julho de 2020. Orientações Educacionais para a Realização de Aulas e Atividades Pedagógicas Presenciais e Não Presenciais no contexto da Pandemia. Ministério da Educação, Conselho Nacional de Educação, Brasília, DF.

Brasil. (2020). Lei $N^{o}$ 14.040, de 18 de agosto de 2020. Estabelece normas educacionais excepcionais a serem adotadas durante o estado de calamidade pública reconhecido pelo Decreto Legislativo nº 6, de 20 de março de 2020, e altera a Lei nº 11.947 de 16 de junho de 2009. Brasília, DF.

Brasil. (2020). Medida Provisória $n^{\circ}$ 936, de $1^{\circ}$ de abril de 2020. Institui o Programa Emergencial de Manutenção do Emprego e da Renda e dispõe sobre medidas trabalhistas complementares para enfrentamento do estado de calamidade pública reconhecido pelo Decreto Legislativo $\mathrm{n}^{\circ} 6$, de 20 de março de 2020 , e da emergência de saúde pública de importância internacional decorrente do coronavírus (covid-19), de que trata a Lei n 13.979 , de 6 de fevereiro de 2020, e dá outras providências. Secretaria Geral, Brasília, DF.

Brasil. (2020). Parecer CNE/CP n 5/2020, de 28 de abril de 2020. Reorganização do Calendário Escolar e da possibilidade de cômputo de atividades não presenciais para fins de cumprimento da carga horária mínima anual, em razão da Pandemia da COVID-19. Ministério da Educação, Conselho Nacional de Educação, Brasília, DF.

Brasil. (2020). Decreto $n^{o} 10.502$ de 30 de setembro de 2020. Institui a Política Nacional de Educação Especial: Equitativa, Inclusiva e com Aprendizado ao Longo da Vida. Brasília, DF.

Brasil. (2015). Lei $n^{\circ}$ 13.146, de 6 de Julho de 2015. Lei Brasileira de Inclusão da Pessoa com Deficiência.

Brasil. (2012). Cartilha do Censo 2010 - Pessoas com Deficiência / Luiza Maria Borges Oliveira / Secretaria de Direitos Humanos da Presidência da República (SDH/PR) / Secretaria Nacional de Promoção dos Direitos da Pessoa com Deficiência (SNPD) / Coordenação-Geral do Sistema de Informações sobre a Pessoa com Deficiência, Brasília.

Brasil. (2009). Decreto $n^{\circ}$ 6.949, de 25 de agosto de 2009. Convenção Internacional sobre os Direitos das Pessoas com Deficiência.

Brasil. (1988). Constituição da República Federativa do Brasil. Brasília, DF: Senado Federal.

Brasil. (1961). Lei n 4.024, de 20 de Dezembro de 1961. Diretrizes e Bases da Educação Nacional.

Costa Carvalho, R. A. C. (2020). Desafios Pedagógicos: antes e na Pandemia COVID 19. Temas em Educ. e Saúde, Araraquara, 16(2), 594-606. https://doi.org/10.26673/tes.v16i2.14061

Coura, A. S., \& Almeida, I. J. (2020). Reflexões sobre a pandemia da COVID-19 e pessoas com deficiência. Journal Health NPEPS, 5(2), 16-19.

Estefenon, S. et al. (2020). Recomendações sobre o uso saudável das telas digitais em tempos de pandemia da COVID-19 \#BOAS TELAS \#MAIS SAÚDE. Sociedade Brasileira de Pediatria.

Matuoka I. (2020). Garantia da educação inclusiva durante a pandemia é direito dos estudantes. Centro de Referência em Educação Integral.

Morgado, J. C., Sousa, J., \& Pacheco, J. A. (2020). Transformações educativas em tempos de pandemia: do confinamento social ao isolamento curricular. Praxis educativa, 15, e2016197, pp. 1-10.

Oliveira, P. de J. D., Oliveira, W. P., \& Barbosa, R. P. C. (2021). The perception of teachers about the inclusion in the remote teaching of students with disabilities during the new coronavirus pandemic. Research, Society and Development, 10(7), e4710716380. https://doi.org/10.33448/rsd-v10i7.16380.

Opas. (2021). Folha informativa COVID-19 - Escritório da OPAS e da OMS no Brasil. OMS - Organização Mundial da Saúde.

Pereira, G., Ortigão, M. I. R. (2016). Pesquisa Quantitativa em educação: algumas considerações. Periferia. 6(1), 66-79.

Souto, X. M. (2020). COVID-19: Aspectos gerais e implicações globais. Revista de Educação, Ciência e Tecnologia de Almenara, 2 (1), 12-36.

Souza, F. F., \& Dainez, D. (2020). Educação especial e inclusiva em tempos de pandemia: o lugar de escola e as condições do ensino remoto emergencial. Práxis Educativa, 15, e2016303. 